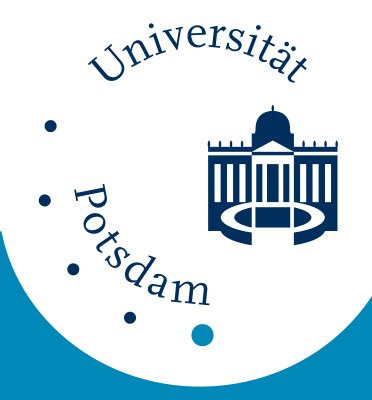

Universität Potsdam

Robert Braun, Fred Feudel, Parvez Guzdar

The route to chaos for a two-dimensional externally driven flow

NLD Preprints ; 46 


\title{
The route to chaos for a two-dimensional externally driven flow
}

\author{
R. Braun, ${ }^{1}$ F. Feudel, ${ }^{1,2}$ and P. Guzdar, ${ }^{2}$ \\ ${ }^{1}$ Institut für Physik, Universität Potsdam, PF 601553, D-14415 Potsdam, Germany \\ ${ }^{2}$ Institute for Plasma Research, University of Maryland, College Park, Maryland 20742
}

We have numerically studied the bifurcations and transition to chaos in a two-dimensional fluid for varying values of the Reynolds number. These investigations have been motivated by experiments in fluids where an array of vortices was driven by an electromotive force. In these experiments, successive changes leading to complex motion of the vortices, due to increased forcing, have been explored [P. Tabeling et al., J Fluid Mech. 213, 511 (1990)]. We model this experiment by means of the two-dimensional Navier-Stokes equations with a special external forcing, driving a linear chain of eight counterrotating vortices, imposing stress-free boundary conditions in the vertical direction and periodic boundary conditions in the horizontal direction. As the strength of the forcing or the Reynolds number is raised the original stationary vortex array becomes unstable and a complex sequence of bifurcations is observed. Several steady states, periodic branches and a period doubling cascade appear on the route to chaos. For increasing values of the Reynolds number, shear flow develops, for which the spatial scale is large compared to the scale of the forcing. Furthermore we have investigated the influence of the aspect ratio of the container as well as the effect of no-slip boundary conditions at the top and bottom, on the bifurcation scenario.

47.20.Ft, 47.27.Cn, 47.54+r

\section{INTRODUCTION}

The transition to chaos from simple laminar flow to chaotic flow is a fascinating phenomenon in nature. Sophisticated experiments, with better diagnostics have been set up to study this transition with the aim to getting a better understanding of the underlying mechanisms. From the theoretical viewpoint, bifurcation theory provides a proper framework to describe and classify these instabilities. In a series of papers, the linear stability of two-dimensional Navier-Stokes flows with different geometry [1-6] has been investigated. The application of bifurcation methods to the NSE, which go beyond a linear stability analysis, is a relative new area of research and hitherto has only been applied to the simplest flows, like the so-called Kolmogorov-flows [7-14]. The objective of the present paper is to apply methods of nonlinear dynamics to investigate the qualitative behavior of the two-dimensional NSE, driven by an external forcing, which can be used as a model for experiments performed by Tabeling et al. [15-18].

In these experiments the transition to turbulence in a linear chain of electrically driven vortices was stud- ied. By increasing the Reynolds number, which was controlled by the strength of the applied current, variation in the spatial structure and temporal evolution of the flow was explored. Motivated by these experiments, numerical calculations of the two-dimensional NSE were performed by Finn et al. and Guzdar et al. in Refs. [19,20]. The formation of large scale shear flow patterns, which could be interpreted as a consequence of the inverse energy cascade in two dimensions, was observed in these theoretical investigations, in agreement with the experiments. Independently, Nakamura has also studied the transition to turbulence for such a linear vortex array but using a slight different model [21]. He imposed noslip conditions for the lateral boundaries and introduced an additional friction term modeling the influence of the bottom. These investigations are aimed as comparing the numerical results with the experimental observations described in Ref. [22]. An analytical approach to model these experiments has been proposed by Dauxois et al. [23] using the Mallier-Maslowe vortex street [24] as an exact solution of the Euler equations. They estimated its stability both for the ideal and for the viscous situations and discussed their results in relation to the experimental observations.

The purpose of the present paper is to continue the study of Guzdar et al. [20] but for the case of eight driven vortices. As a model we have used the twodimensional NSE with periodic boundary conditions in horizontal direction and stress-free boundary condition in vertical direction. It should be noted that the idealized two-dimensional model cannot capture all details of the experiment mentioned above, but it can demonstrate typical features of fluid dynamics on its route to chaos. The goal of the present paper is to examine the qualitative changes employing special bifurcation techniques. In Sec. II we introduce the basic equations and explain the forcing term, modeling the Lorentz force applied to the electrolyte in the experiments. Then, in Sec. III we start our investigations imposing stress-free boundary conditions at the top and bottom. We present the bifurcation scenario on the route to chaos and describe the corresponding qualitative changes of the flow. In Sec. IV we study the variations of the bifurcation structure and the resulting dynamics, using no-slip boundary conditions instead of the free-slip conditions. Finally, Sec. V gives a short discussion about the practical importance of the theoretical results and provides arguments for the necessity to extend the model to three dimensions. 


\section{BASIC EQUATIONS AND NUMERICAL METHODS}

To model the experiment of Tabeling we have used the two-dimensional NSE with an external forcing which imitates the Lorentz force due to the current flowing through the electrolyte in a plexi-glass cell with an array of alternating north and south pole magnets at the bottom of the cell. We start with the incompressible NSE in the rescaled form

$$
\begin{gathered}
\frac{\partial \mathrm{v}}{\partial t}+(\mathrm{v} \cdot \mathrm{r}) \mathrm{v}=\nabla^{2} \mathrm{v}-\mathrm{r} p+\mathrm{f} \\
\mathrm{r} \cdot \mathrm{v}=0
\end{gathered}
$$

where $\mathrm{v}$ is the fluid velocity field, $p$ is the thermal pressure and $\mathrm{v}$ represents the external force which is chosen to be

$$
\mathrm{f}=f\left(\begin{array}{r}
\sin k_{1} x \cos k_{2} y \\
-\cos k_{1} x \sin k_{2} y
\end{array}\right)
$$

To obtain the NSE in the nondimensional form of Eqs. (1-3) a rescaling transformation to the typical length and time scales, which can be found explicitly in Ref. [14], has been applied. The two constants $k_{1}$ and $k_{2}$ in Eq. (3) are fixed to $k_{1}=8 \pi / L_{x}$ and $k_{2}=\pi / L_{y}$ thereby driving an array of eight counterrotating eddies on the set $\Omega=\left[L_{x}, L_{y}\right]$. By means of a further rescaling transformation the length $L_{y}$ can be normalized to $\pi$ and there remain only two free parameters in the equations. They are the horizontal length $L_{x}$ and the strength of the forcing $f$ which is in turn related to the Reynolds number. For weak forcing, the term $\mathrm{f}$ in Eq. (1) can be evaluated to be equal to the Laplacian term. Hence the Reynolds number can be estimated to be $R e \approx f$. For strong forcing, on the other hand, this term is approximately balanced by the convective term and it turns out that $R e \approx f^{1 / 2}$ [25]. For the sake of simplicity we consider periodic boundary conditions for the velocity field in the horizontal direction, $\mathrm{v}(x, t)=\mathrm{v}\left(x+L_{x}, t\right)$, but impose both stress-free and no-slip boundary conditions at top and bottom of the two-dimensional fluid with the aim to study their influence on the bifurcations.

In our numerical computations we did not directly integrate Eq. (1) but rather the corresponding equation for the vorticity $!=\mathrm{r} \times \mathrm{v}$. By restriction to two spatial dimensions, this equation reduces to one scalar equation for the only nonvanishing component $\omega=\omega_{z}$ of the vorticity:

$$
\frac{\partial \omega}{\partial t}+(\mathrm{v} \cdot \mathrm{r}) \omega=\nabla^{2} \omega+f\left(k_{1}+k_{2}\right) \sin k_{1} x \sin k_{2} y
$$

Successive bifurcations of an array of counterrotating vortices, produced by the external forcing in the form of Eq. (3), will be analyzed by changing the scalar control parameter $f$. This is the essential bifurcation parameter in our studies. In other words we will study the transition from a simple motion to chaotic dynamics of the fluid for increasing values of the Reynolds numbers, both for stress-free and no-slip boundary conditions, as well as for different aspect ratios of the driven cells, determined by the second free parameter $L_{x}$.

For the case of stress-free boundary conditions

$$
\left.\frac{\partial v_{x}}{\partial y}\right|_{y=y_{0}}=0, \quad v_{y}\left(x, y_{0}\right)=0 \quad \text { for } \quad y_{0}=0, L_{y}
$$

which in turn implies $\omega\left(x, y_{0}, t\right)=0$, we have used a pseudospectral code based on a Fourier expansion of the vorticity in the form of

$\omega=\sum_{k_{x}, k_{y} \in \mathbb{Z}} \hat{\omega} \mathrm{k}(t) e^{i k_{x} x} \sin \left(k_{y} y\right), \quad \mathrm{k}=\left(2 \pi k_{x} / L_{x}, 2 \pi k_{y} / L_{y}\right)$.

Inserting this Fourier ansatz into Eq. (4) leads to an infinite system of ordinary differential equations for the real and complex components of the Fourier components $\hat{\omega}$, which has been studied numerically by a finitedimensional truncation using a pseudospectral code with a resolution of $64 \times 32$ gridpoints.

Beside the pseudospectral code we have used a finite difference code with the same resolution, mainly to study the dynamics for the no-slip conditions, but also to crosscheck the results for the stress-free boundary conditions for which the finite-difference code can be easily modified.

\section{BIFURCATION SCENARIO FOR STRESS-FREE BOUNDARY CONDITIONS}

In this section we present a detailed bifurcation analysis of Eq. (4) for the case of stress-free boundary conditions in the transverse direction and for a fixed aspect ratio given by the conditions, $L_{y}=\pi$ and $L_{x}=4 \pi$. To find out the qualitative behavior of the solutions we have applied bifurcation techniques on the truncated finite dimensional system of ODEs for the Fourier coefficients $\hat{\omega} \mathrm{k}$. For instance, the steady state branches have been traced as a function of the bifurcation parameter $f$ by means of a special continuation program. Simultaneously the eigenvalues of the Jacobian matrix were calculated in order to detect bifurcations, when some eigenvalues cross the imaginary axis.

For a small forcing, i.e. for small values of $f$, the array of eight counterrotating vortices (see Fig. 1a)) is the only time-asymptotic state. Here only the forced modes are excited and the solution of Eq. (4) can be expressed analytically in the form of

$$
\omega(x, y)=\frac{f\left(k_{1}+k_{2}\right)}{k_{1}^{2}+k_{2}^{2}} \sin \left(k_{1} x\right) \sin \left(k_{2} y\right) .
$$

Increasing the strength of the forcing, this primary steady state loses its stability producing a secondary steady flow 
with a shear component. The formation of the shear flow is already described in detail by Guzdar et al. for the case of four driven vortices [20]. This secondary steady state branch consists of four co-rotating vortices and a shear component, which are separated by heteroclinic lines connecting the fixed points of the flow (c.f. Fig. 1b)).
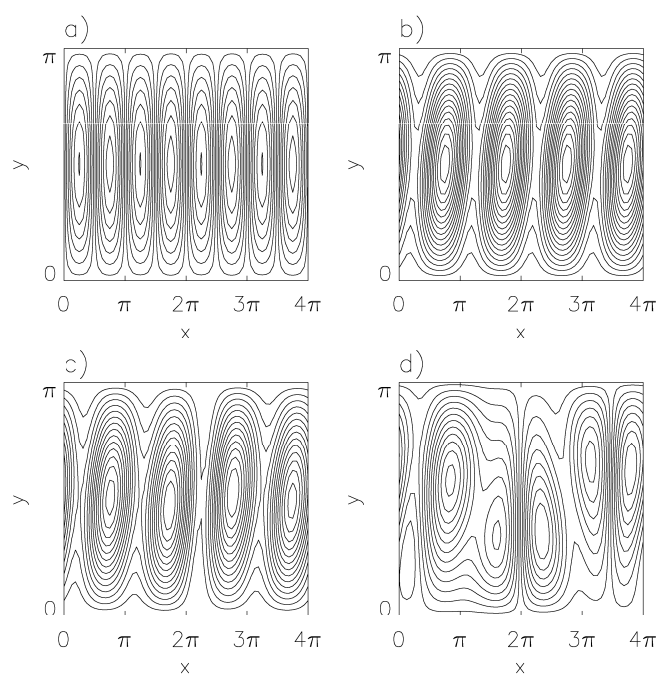

FIG. 1. Streamlines of the steady state branches: a) Steady I $(f=26.7)$, b) Steady II $(f=43.3)$, c) Steady III $(f=44.7), \mathrm{d})$ Steady IV $(f=100.0)$.

Tracing this secondary steady branch a further pitchfork bifurcation takes place. It creates tertiary steady states, for which the shape of the flow is changed such, that the middle points of neighbouring co-rotating vortices are shifted up and down. This branch is only stable within a small interval of the bifurcation paramter $f$ and is finally terminated by a Hopf bifurcation.

A complete overview on all solution branches, which we have found, are compiled in Tab. I and are presented schematically in Fig. 2.

\begin{tabular}{lll}
\hline \hline Branch & Stability interval & Remerks \\
\hline Steady I & $f<29.7$ & 8 counterrotating vortices \\
Steady II & $29.7<f<43.7$ & 4 co-rotating vortices \\
Steady III & $43.7<f<45.7$ & 4 tilted vortices \\
Period I & $45.7<f<54.7$ & created by a Hopf point \\
Steady IV & $47.7<f<106$ & \\
Period II & $106<f<227$ & \\
Period doubling & $227<f<240$ & \\
Chaos & $240<f$ & \\
\hline \hline
\end{tabular}

TABLE I. The different solution branches.

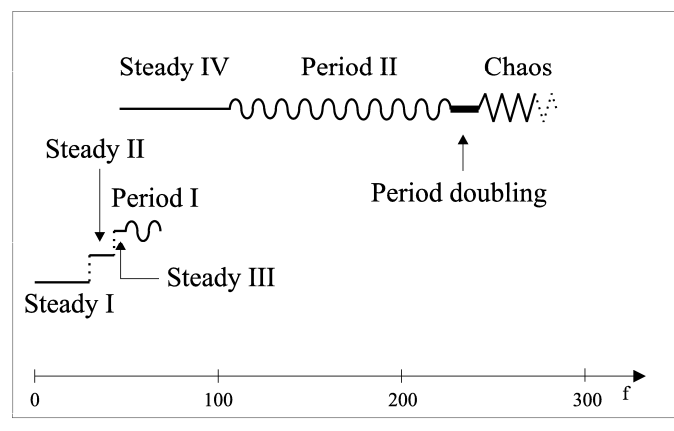

FIG. 2. Schematic bifurcation diagram

The three steady state branches described above are labelled here as Steady I, Steady II and Steady III, respectively.

The Hopf bifurcation at Steady III produces a periodic solution branch Period I, for which the streamlines are subject to a complex reconnection process. In Figs. 3a)3f) the contours of the streamline portrait are plotted at a few instants of time during a period. This helps to visualize the time evolution of the velocity field. For most of the time, the pattern consists of two pairs of combined eddies which are separated by slender vortices as presented in Fig. 3a) and Fig. 3f). A rapid reconnection process, demonstrated in Figs. 3b)-3e), transforms both of the states, quasi-stable in time, into one another.
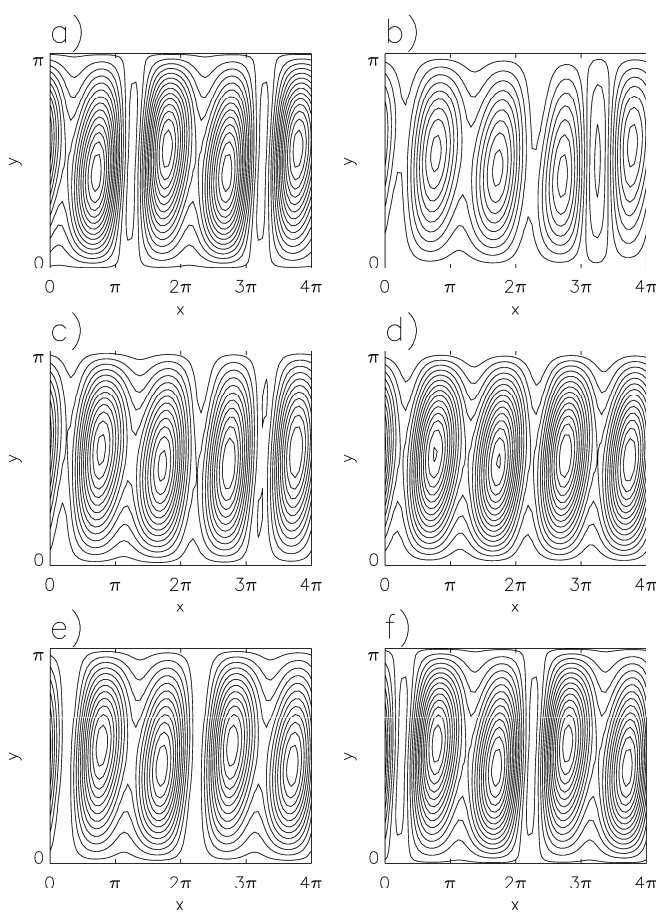

FIG. 3. Snapshots of streamlines belonging to a periodic solution (Period I) at different time points.

For increasing Reynolds number the basin of attraction of this periodic branch shrinks and finally disappears for $f=54.7$. 
As seen in Fig. 2, for higher Reynolds number, another branch dominates the dynamics of the system. So we have found a further steady state (Steady IV), partly coexisting with the periodic branch Period I. Its streamlines are drawn in Fig. 1d). This steady state ends up again in a Hopf bifurcation creating the periodic branch Period II, which in turn undergoes a period doubling cascade leading finally to chaos. In Figs. 4a)-4c) the projection of the trajectory onto the real parts of certain Fourier modes are depicted for the original periodic orbit and for the orbits after the first two period doublings. The final chaotic attractor, projected in this sub-space, is shown in Fig. 4d).
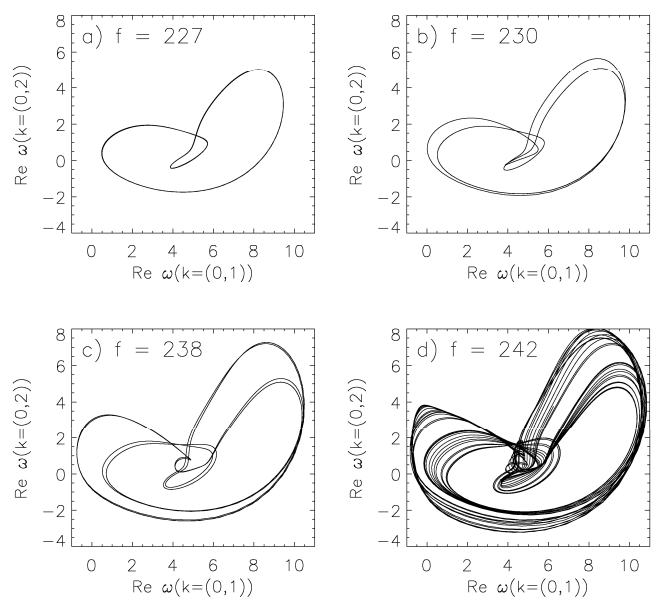

FIG. 4. Projection of the trajectory onto the real parts of the mode $\mathrm{k}=(0,1)$ and $\mathrm{k}=(0,2)$ in the period doubling cascade on the route to chaos for (a) periodic orbit (Period II), (b) orbit after first period doubling, (c) orbit after second period doubling $(\mathrm{d})$ chaotic attractor (Chaos).

To verify that the state is truly chaotic, we have calculated the largest Lyapunov exponents for selected values of the bifurcation parameter, using an algorithm developed by Shimada and Nagashima [26]. Fig. 5 shows for $f=245$ the cumulative values of the five largest Lyapunov exponents as a function of the integration time. It demonstrates the good convergence of the algorthm and reveals that one of the expontents is positive. This proves that for this value of the forcing parameter, the state is indeed chaotic.



FIG. 5. The five largest Lyapunov exponents versus integration time for $f=245$.

The streamline portraits of the upper branches of the bifurcation diagram in Fig. 2 are much the same and the structure of the corresponding velocity fields are comparable to that of Steady IV as in Fig. 1d), varying only weakly in time, even for the chaotic state.

To study the influence of the aspect ratio on the bifurcation we have modified the horizontal length $L_{x}$ of the box to $3 \pi$ and to $5 \pi$. In both cases we have found the same bifurcation scenario as described above. We conclude, that the bifurcation structure is relatively insensitive to the changes in the aspect ratio. Only the bifurcation points are shifted to higher values of the forcing parameter for a smaller value of $L_{x}$ and they shift to lower values of the forcing parameter for larger values of $L_{x}$.

\section{COMPARISON WITH NO-SLIP BOUNDARY CONDITIONS}

In this section we report on the bifurcations when noslip boundary conditions are imposed at the top and bottom of our computation box,

$$
\mathrm{v}\left(x, y_{0}, t\right)=0 \quad \text { for } \quad y_{0}=0, L_{y}
$$

The lengths of the computation box have been chosen, as in the previous section, to be $L_{x}=4 \pi$ and $L_{y}=\pi$, respectively. We are interested in the influence of the boundary conditions on the bifurcation behavior in general, even though the boundary conditions that have been realized in the experiments, performed by Tabeling et al., are nearly stress-free [16].

For a small forcing strength the only stable solution, satisfying the no-slip boundary conditions, is again the flow consisting of eight counterrotating vortices (c.f. Fig 6a)). 

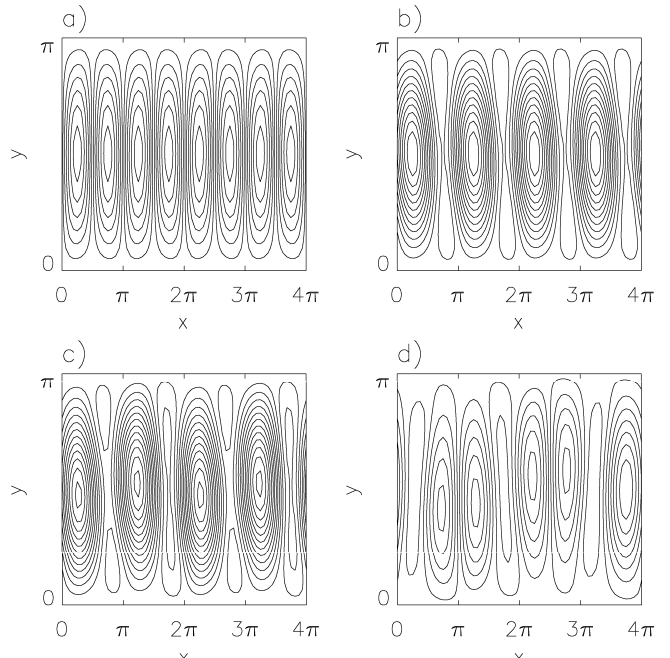

FIG. 6. Streamlines of the steady state branchse: a) primary steady state $(f=100), b)$ secondary steady state $(f=170)$, c) tertiary steady state $(f=180), \mathrm{d})$ additional steady state $(f=250)$.

In contrast to the the case of stress-free boundary conditions, the no-slip conditions have a stabilizing effect on this primary steady state, which loses its stability for a much higher value of the forcing parameter, $f=138$. In the vicinity of the bifurcation point the changes of the streamlines are very similar to the stress-free boundary conditions and, as described in more detail by Guzdar et al. [20], every alternate vortex is compressed compared to its neighbour. But due to the no-slip boundary conditions no shear flow has been created and the secondary steady state branch finally settles down to a streamline portrait very similiar to the one presented in Fig. 6b). Tracing this branch for larger forcing, a further pitchfork bifurcation leads to a tertiary steady state for which the center points of neighbouring vortices are shifted into opposite directions away from the horizontal center line of the fluid region (6c). Again the situation is analogous to that of Steady III in Tab. I. This branch in turn ends up in a Hopf bifurcation at $f=188$ producing a periodic solution.

It is not the intention of this section to determine all the bifurcation points exactly but just to study the qualitative behavior of different bifurcations in comparison to the case of stress-free boundary conditions. Similar to the bifurcation diagram in Fig. 2 the periodic branch is only stable for a certain interval of the forcing parameter and another steady state, at first coexisting with the periodic solution and finally forming the global attractor, appears. The corresponding streamline portrait is presented in Fig. 6d). This branch is comparable with the upper branch (Steady IV) in Fig. 2 but its solutions doens't show the dominating large scales of a shear flow as in the case of stress-free boundary conditions.

For increasing values of the the strength of the forcing, qualitative changes of the flow occur, which are different from those bifurcations observed in the case of stress-free boundary conditions. In Figs. 7a) - e) the time history at one spatial location point is plotted with the aim to identifying and classifying the different dynamical regimes.
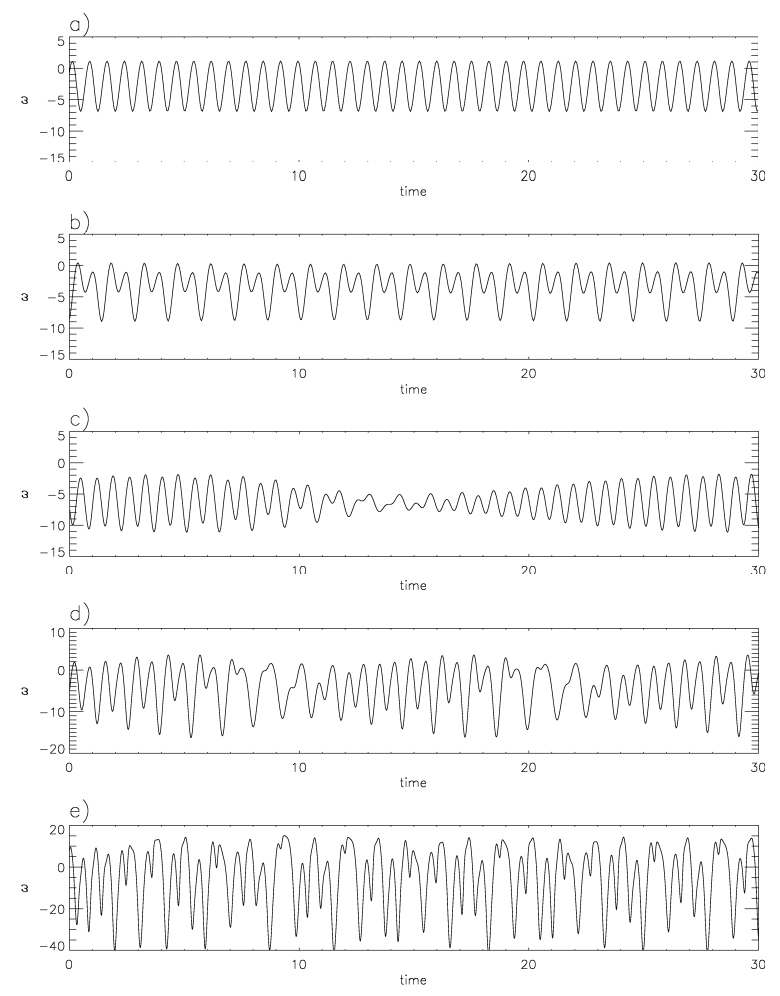

FIG. 7. Time history of the vorticity at a spatial location point $x=0, y=\pi / 2$ for a) $f=277$, b) $f=282$, c) $f=287$, d) $f=296$ and e) $f=350$.

The first bifurcation, namely a Hopf bifurcation leading to a periodic solution, c.f. Fig. 7a), which is followed by a period doubling bifurcation, presented in Fig. 7b), are still the same as in the scenario described in the previous section. But for larger values of the forcing parameter a large time scale modulation of the amplitude, first observed at $f=287$ in Fig. 7 c), has an essential influence on the subsequent nature of the route to chaos. This was not observed in the no-slip case. The slow modulation frequency could be a result of a secondary Hopf bifurcation, but might also be a consequence of a degenerate bifurcation, producing traveling wave-like solutions. By a further increase of the forcing strength this second frequency becomes a bit larger and the time history of the amplitude looks much more like a generic torus solution (c.f. Fig. 7d)). As seen in Fig. 7e), the motion becomes eventually more and more irregular and we conjecture a transition to chaos via a torus destruction, another well-known route to chaos $[27,28]$. But the final proof of the chaotic nature of the attractor, by calculation of the largest Lyapunov exponent at present remains work for the future. We are going to develop a separate code using Chebyshev polynomial expansions in the vertical direction for a better treatment of the no-slip boundary 
conditions. This will allow us to cross-check the resulting dynamics with those obtained from our finite-difference code and will also allow us to compare the routes to chaos with the two codes.

\section{DISCUSSION AND CONCLUSION}

Motivated by experiments of Tabeling et al. [15-18] we have investigated the bifurcation scenario for a chain of eight externally driven vortices in a two-dimensional Navier-Stokes fluid by increasing the strength of the forcing, which corresponds to an increase of the Reynolds number. According to the experimental set-up stress-free boundary conditions at the top and bottom are appropriate to model this experiment and in our studies, we have mainly focussed on this case. We found a complex bifurcation sequence including a period doubling cascade, which leads finally to a temporally chaotic motion of the velocity field. A direct comparison of the numerical results with the experiments is only possible for the first pitchfork bifurcations. The bifurcations experimentally observed for higher Reynolds numbers depend strongly on the thickness of the fluid layer, and this three-dimensional effect can't be modelled by the twodimensional NSE used here. For a more extensive discussion we give a reference to a companion paper in Ref. [20]. Other essential features, as the appearance of large scale flows dominating the dynamics at higher Reynolds number, are in good agreement with the observations. Also for various magnet configurations (four, six and eight), for large forcing, the experiments of Tabeling et. al have shown a clear indication of the period-doubling (subharmonic) route to chaos, c.f. [16]. To get a more detailed agreement between experiment and numerical bifurcation analysis, both the three-dimensional effects as well as different boundary condition in the direction of the array of vortices need to be considered. We have assumed a periodic boundary condition. However in the actual experiment, the region beyond the driven vortices is open. In preliminary investigations in studying the influence of the bottom friction we showed that this stabilizing effect shifts the bifurcations to higher values of the Reynolds number but the qualitative features of the dynamics survive, at least for moderate Reynolds numbers [29]. We conclude with this discussion that even with our very idealised simulations we have captured many interesting features observed in the experiments.

The influence of no-slip boundary conditions on the bifurcations are, in our opinion, of general interest. We demonstrated that, at least for the two-dimensional Navier-Stokes flow considered here, the bifurcation scenario is relatively robust with respect to variations of the imposed boundary conditions. As expected, the no-slip boundary conditions have, in comparison to stress-free boundary conditions, a stabilizing effect. Up to the first period doubling, c.f. Fig. 2, we recover the same bifur- cations for no-slip boundary conditions as we did for the free-slip case. Only the final route to chaos deviates. In contrast to the period doubling cascade, observed for stress-free boundary conditions, a second frequency appears for the no-slip case and the chaotic motion in this case is seemingly a result of a destruction of the twofrequency torus.

[1] G. I. Shivashinsky and V. Yakhat, Phys. Fluids 28, 1040 (1985).

[2] K. Gotoh and Y. Yamada, J. Phys. Soc. Jap. 53, 3395 (1984).

[3] K. Gotoh and Y. Yamada, Fluid Dyn. Res. 1, 165 (1986).

[4] A. Thess, Phys. Fluids A 4, 1396 (1992).

[5] A. Thess, Phys. Fluids A 5, 335 (1993).

[6] H. Fukuta and J. Murakami, J. Phys. Soc. Japan 65, 1655 (1996).

[7] C. Boldrighini and V. Franceschini, Commun. Math. Phys. 64, 159 (1979).

[8] V. Franceschini and C. Tebaldi, J. Stat. Phys. 21, 707 (1979).

[9] V. Franceschini, C. Giberti, and M. Nicolini, J. Stat. Phys. 50, 879 (1988).

[10] V. Franceschini and C. Giberti, Theor. Comput. Fluid Dyn. 2, 185 (1991)

[11] J. Lee, Chaos 2, 537 (1992).

[12] M. S. Jolly, Physica D 63, 8 (1993).

[13] F. Feudel and N. Seehafer, Chaos, Solitons \& Fractals 5, 1805 (1995).

[14] F. Feudel and N. Seehafer, Phys. Rev. E 52, 3506 (1995).

[15] P. Tabeling, B. Perrin, and S. Fauve, Europhys. Lett. 3, 459 (1987).

[16] P. Tabeling, O. Cardoso, and B. Perrin, J. Fluid Mech. 213, 511 (1990).

[17] O. Cardoso, H. Willaime, and P. Tabeling, Phys. Rev. Lett. 65, 1869 (1990).

[18] H. Willaime, O. Caedoso, and P. Tabeling, Phys. Rev. E 48, 288 (1993).

[19] J. M. Finn, J. F. Drake, and P. N. Guzdar, Phys. Fluids B 4, 2758 (1992).

[20] P. N. Guzdar, J. M. Finn, A. V. Rogalsky, and J. F. Drake, Phys. Rev. E 49, 2062 (1994).

[21] Y. Nakamura, J. Phys. Soc. Japan 65, 1666 (1996).

[22] H. Honji, in Proc. the Sixth Asian Congress of Fluid Mech., edited by Y. T. Chew and T. P. Tso (Nanyang Technological University, Singapure, 1995), p. 1218.

[23] T. Dauxois, S. Fauve, and L. Tuckerman, Phys. Fluids 8, 487 (1996).

[24] R. Mallier and S. A. Maslowe, Phys. Fluids A 5, 1074 (1996).

[25] B. Braun, F. Feudel, and N. Seehafer, Phys. Rev. E 55, 6979 (1997).

[26] I. Shimada and T. Nagashima, Progr. Theor. Phys. 61, 1605 (1979).

[27] S. Newhouse, D. Ruelle, and F. Takens, Comm. Math 
Phys. 64, 35 (1978).

[28] V. Franceschini and C. Tebaldi, Commun. Math. Phys.

94, 317 (1984).

[29] R. Braun, Ph.D. thesis, University of Potsdam, 1997. 